\title{
Why do Generative Learning Strategy Improve Memory in VR? - Based on ICALM
}

\author{
Wenya Yang and Xue Wang
}

\begin{abstract}
Generative learning strategies interconnect with cognition and emotion. Based on one-factorial experimental design, 75 participants were randomly assigned to study a chemical Virtual Reality (VR) lesson in one of three conditions: VR, VR+ summarizing, and VR+ self-testing. An emWave system was used to record the learners' emotional state during learning. The learners' learning outcomes were measured with retention tests, learning experiences were measured with instruments. The results showed that compared to the students were given a VR lesson without generative learning strategy, 1) the students who engaged in generative self-testing strategy during learning displayed more positive emotions in the cognition process, more positive ratings after learning, and higher memory test scores; 2) the students who engaged in generative summary strategy during learning showed more positive emotions in the cognition process, but lower immediate memory scores. These findings give new evidence to explaining how generative summarizing and self-testing learning strategies affect learning based VR.
\end{abstract}

Index Terms-Emotion, generative learning strategy, VR.

\section{INTRODUCTION}

Virtual Reality (VR) has three characteristics (i.e., immersion, interaction, and imagination) in simulating three-dimensional (3D) virtual world with the help of computer. According to the immersive level and application scenarios, the types of VR can be divided into Desktop VR, Immersive VR, Augmented VR, and Distributed VR. VR have become a very popular way to teach learners in a wide range of subjects, which enhance the learner's perception of all kinds of information with its unique teaching situation. Therefore, the question of how to effectively learn from VR has drew education researchers' attention.

Many researchers underlined that VR technology in teaching made learners have a better learning experience, such as, learning motivation, interests, flow experience and so on. For example, Shih examined the effects of three-dimensional virtual environment on London's culture learning, the results showed that all learners benefitted from cultural immersion [1]; Zhou stressed that the use of VR-supported learning application not only made the learning interesting, but also promoted the construction of

Manuscript received March 9, 2021; revised July 5, 2021. This work was supported in part by MOE (Ministry of Education in China) Project of Humanities and Social Sciences (foundation no. 19YJC880090) and Key Cultivation Projects of Tianjin Teaching Achievement Award (foundation no. PYGJ-014).

The authors are with the Faculty of Education, Tianjin Normal University, Tianjin, China (e-mail: YYYang_A@163.com, wangxuetjnu@qq.com). knowledge in practices [2]; Makransky highlighted that application of VR in undergraduate course of medical genetics improved the motivation level of learners [3] ; Cheng underlined that VR guided undergraduates' learning role identity from passive to active [4].

However, prior research investigating VR has shown no or negative influence for learning effect. For example, Parong stressed that the immediate learning effect of PPT learners was significantly better than that of VR learners in biology [5]; Leder highlight that compared with PowerPoint teaching, the application of VR did not significantly improve the learning effect of learners in safety training teaching [6]; Makransky compared the immersive VR and Desktop VR, stressed that there was no significant difference in the immediate test of undergraduate in the course of laboratory safety training [7].

Mayer, the founder of multimedia learning research, underlined that it was not the technology itself that triggered and promoted learning, but the best strategies and methods that matched it. Meyer proved it, a sample of 118 participants was randomly assigned to one of two media conditions (immersive VR vs video), and one of two method conditions (with pre-training vs without pre-training), the results showed an interaction between media and method on knowledge, transfer, and self-efficacy [8]. It can be seen that it is particularly important to apply tools or techniques in accordance with teaching design.

Learners who learn in VR are not necessarily actively making sense of the learning material via generative learning process where learners are encouraged to engage actively in generating processing - by selecting important information which extremely relevant, organizing it into mental representation in working memory, then integrating it with prior knowledge into a coherent mental model stored into long-term memory. In other word, learners who learning with VR alone are not encouraged to meaningful learning absolutely.

Generative learning strategies always help learners do to foster these meaningful learning behaviors. Generative learning strategies grounded in Generative Learning Theory and Cognitive Theory of Multimedia Learning (CTML), which include eight learning strategies: summarizing, mapping, drawing, imagining, self-testing, self-explaining, teaching, and enacting [9].

Generative summarizing and self-testing strategies need not be trained in advance, and used easily and widely. The core difference between them is whether the question cues are involved, such as teacher verbal questions. The summarizing strategy encourages learners explain the main ideas from what they have learned in their own words. In a recent study, college students viewed instruction video about 
a human cell. The results showed that the application of summarizing strategy can significantly reduce learners' mental effort and improve their cognitive process [10]. The self-testing strategy also referred to as the retrieval-based learning, which asks learners to answer questions about previously learned material. Using this strategy, students' long-term memory of device troubleshooting skills effectively promoted [11]. Many researchers focus on the cognition process and learning outcomes but less on emotion.

However, emotions are inherently interconnected with cognitions. Plass 's Integrated Cognitive-Affective Model of Learning with Multimedia (ICALM, see Fig.1) grounded in the core affect (Russell and Izard) and the Cognitive Affective Theory of Learning with Media (Moreno and Mayer) [12]. As same as Mayer's S-O-I multimedia learning model consisted of selection, organization, and integration, but it stresses that emotions are inherently motivational and interconnected with cognition. The "core affect" means the affective responses that students experience when interacting with a multimedia environment (i.e., using learning strategy to perceive VR information). The "core affect" may be attributed to a specific source, always impacts the selection process of cognition, and then, with the interrelated with interest and motivation appraised by learners, impact the organization of mental representations in working memory, also being by what is processed. The integration of mental representation stored into long-term memory, which included verbal, visual mental representation, and experienced emotions.

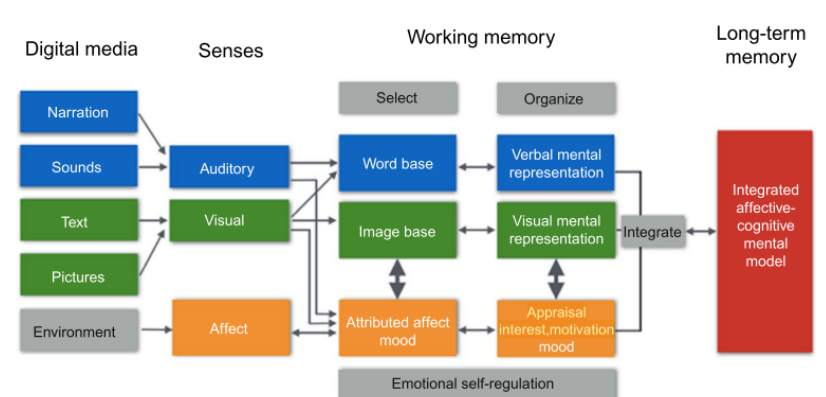

Fig. 1. Integrated cognitive affective model of learning with multimedia (ICALM).

In the current study, we tested the effects of two generative learning strategies (summarizing and self-testing), with the effects being measured in terms of recognition technology while viewing VR lesson; self-reported emotional self-regulation (motivation, positive mood, and negative mood) after the VR lesson; and learning performance (immediate retention and delay-retention).

We adopted the ICALM to inform our hypotheses about learning through generative learning strategies in VR. We posed the following hypotheses.

Hypothesis 1: The generative strategy will induce more emotions in the cognition process than viewing a continuous VR lesson.

Hypothesis 2: Adding a generative strategy prompt to a VR lesson will increase positive ratings toward emotional self-regulation.

Hypothesis 3: Students will have better learning outcomes when they use a generative strategy than viewing a VR lesson without it.

\section{METHOD}

\section{A. Participants and Design}

There were 75 undergraduate students enrolled in Tianjin Normal University. The generative strategy condition was a between-subjects variable. Participants were assigned randomly to the VR (VR group), the VR plus summarizing ( $\mathrm{VR}+\mathrm{S}$ group), or the $\mathrm{VR}$ plus self-testing ( $\mathrm{VR}+\mathrm{ST}$ group). A set of preliminary analyses revealed there were no differences in prior-knowledge, $F=0.66, p=0.937>0.05$.

\section{B. Materials and Apparatus}

The instructional materials consisted of lessons on chemical structure isomerism in VR simulation and a questionnaire used in the VR+ST group. The VR lesson lasted approximately $5 \mathrm{~min}$, which contained narration and immersive animations of the molecular Structural Model. The questionnaire consisted of 4 questions based on the VR lesson and the question frame for teaching.

The paper-based materials consisted of a pre-questionnaire, a post-questionnaire, and two posttests. The pre-questionnaire solicited demographic information, assessed emotional state (PANAS scale [13], Cronbach's $\alpha=0.921$ for PA, 0.948 for NA), and tested prior-knowledge. The post-questionnaire assessed emotional state (PANAS scale), interest and motivation [14] (Cronbach's $\alpha=0.847$ ). The two posttests were a retention test and a delay-retention test. The retention test consisted of 6 multiple-choice questions, and the delay-retention test the same as it. To avoid memory retention, we changed the order of questions.

Using emWave Pro (6030) which identify human heart rate power spectral density, we can directly notice emotional states [15]. Three color-rendered indexes that represented negative emotions (red part, the low-frequency zone of the power spectral density), neutral emotions (blue part, the medium-frequency zone of the power spectral density), and positive emotions (green part, the high-frequency zone of the power spectral density), respectively, Fig. 2.

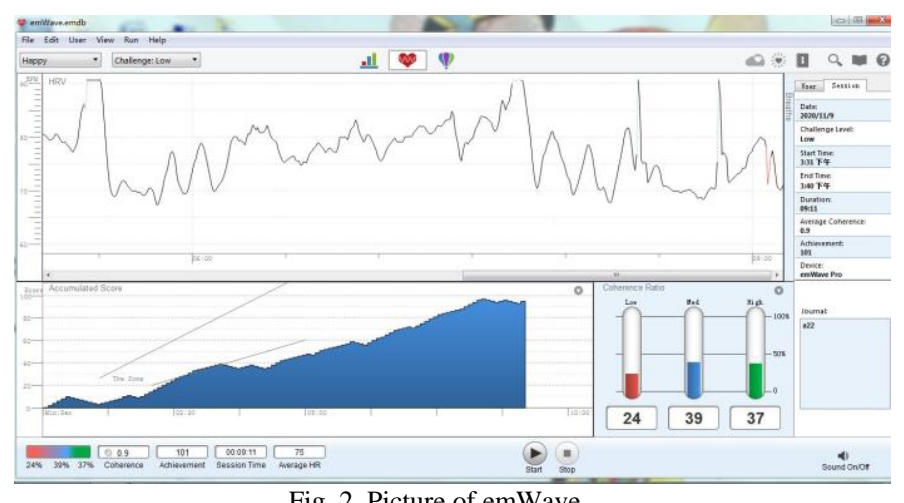

Fig. 2. Picture of emWave

\section{Procedures}

After finishing the pre-questionnaire, all participants got knowledge from VR, and during this process, they wore an emWave earplug that collected emotion data. In the $\mathrm{VR}+\mathrm{S}$ group and the VR+ ST group, participants followed a similar procedure as the VR group, except that they viewed the lesson in 4 segments (the pause time of video is 1 minute 16 seconds, 3 minute 30 seconds, 4 minute 12 seconds, and 5 
minute 16 seconds, respectively).

After watching each segment, the $\mathrm{VR}+\mathrm{S}$ group wrote a summarization of the segment just viewed on a paper, and the $\mathrm{VR}+\mathrm{ST}$ group answered a question of the segment just viewed the same as the $\mathrm{VR}+\mathrm{S}$ group, on a paper.

After finishing the assigned unit, all participants answered the post-questionnaire and retention test immediately; and finally, they would answer the delay-retention test after one week.

\section{RESULT}

A. Does Generative Strategy Induce Emotions in the Cognition Process Than Viewing a Continuous VR Lesson?

Table I shows the mean score on the emotional state in the cognition process for the three groups. Independent Samples T-test illustrated that the VR+ST group induced less negative emotion $(p=0.026<0.05)$ and more positive emotion $(p=0.005<0.05)$ than the VR group significantly, but no significant difference on neutral emotion $(p=0.468>0.05)$. There was more positive emotion $(p=0.038<0.05)$ in the $\mathrm{VR}+\mathrm{S}$ group significantly than the VR group, but no difference on neutral emotion $(p=0.925>0.05)$; and less negative emotion $(p=0.05)$ induced significant marginally. Overall, both of the strategies arose positive emotion in the cognition process.

We conclude that generative strategy induces more positive emotion in the cognition process than viewing a continuous VR lesson, hypothesis 1 is true.

TABLE I: THE MEAN SCORE ON EMOTION IN THE COGNITION PROCESS, EMOTIONAL SELF-REGULATION AND POSTTEST

\begin{tabular}{llll}
\hline \hline & & Group VR & Group VR+Summary \\
\hline & Negative Emotion & $43.44 \pm 18.84$ & $34.28 \pm 12.68$ \\
Emotion in the cognition process & Neutral Emotion & $26.48 \pm 10.38$ & $26.72 \pm 7.28$ \\
& Positive Emotion & $30.08 \pm 15.38$ & $39.00 \pm 14.20$ \\
& Interest & $22.00 \pm 3.00$ & $20.20 \pm 5.00$ \\
& Motivation & $4.92 \pm 1.50$ & $5.04 \pm 1.46$ \\
Emotional & Positive Mood & $29.24 \pm 4.47$ & $29.16 \pm 6.67$ \\
Self-regulation & Negative Mood & $13.64 \pm 4.77$ & $13.76 \pm 6.00$ \\
& Retention test & $9.88 \pm 2.73$ & $8.20 \pm 2.47$ \\
Posttest & Delay-retention test & $8.60 \pm 3.32$ & $7.24 \pm 2.44$ \\
&
\end{tabular}

Note: To avoid the confusion of the name of the emotional states when they between "Emotion in the cognition process" and "Emotional Self-regulation", we called the positive emotional state "positive mood" and the negative emotional state "negative mood" respectively in Emotional Self-regulation.

\section{B. Does Add Generative Strategy Prompts to a VR} Lesson Increase Positive Ratings toward Emotional Self-regulation?

Table 1 also shows the mean rating on the emotional self-regulation for the three groups. Independent Samples T-test demonstrated that the $\mathrm{VR}+\mathrm{ST}$ group enhanced academic motivation $(p=0.01<0.05)$ significantly, but no significantly difference on ratings of interest $(p=0.458>0.05)$, positive mood $(p=0.346>0.05)$, and negative mood $(p=0.745>0.05)$. It was also revealed that the $\mathrm{VR}+\mathrm{S}$ group did not give significantly different ratings on interest $(p=0.129<0.05)$, motivation $(p=0.775>0.05)$, positive mood $(p=0.960>0.05)$, and negative $\operatorname{mood}(p=0.938>0.05)$ after the lesson. In other words, adding the Self-Testing strategy prompts to a VR lesson could increase positive ratings toward the lesson, but Summarization could not.

We conclude that adding generative strategy prompts to a VR lesson increase positive ratings toward emotional self-regulation, hypothesis 2 is true.

\section{Do Students Have Better Learning Outcomes Than} Viewing a VR Lesson without a Generative Strategy?

Table I still shows the mean score on the posttest for the three groups. Independent Samples T-test revealed that the $\mathrm{VR}+\mathrm{ST}$ group performed significantly better than the VR group on the immediate retention test $(p=0.046<0.05)$ and delay-retention test $(p=0.044<0.05)$. The $\mathrm{VR}+\mathrm{S}$ group and the VR group was no difference in the delay-retention test $(p=0.105>0.05)$ but a significant difference in the immediate retention test $(p=0.027<0.05)$. In short, students learned more from a chemical lesson presented in VR plus the Self-Testing strategy, but less in VR plus Summary strategy.

We conclude that students have better learning outcomes than viewing a VR lesson without the generative Self-Testing strategy, hypothesis 3 is true.

\section{DISCUSSION}

\section{A. Empirical Contributions}

Students have better performance on the immediate retention tests and delay-retention tests when using a Self-Testing strategy in VR than without it. The questions that learners answered reduced the difficulty of learning material perception, because it has a strong role of learning orientation and scaffolding, which increases the learners' motivation in the process of learning material information selection, organization and integration. The experimental data also prove it.

However, we found that the immediate retention tests decreased when learners use a Summary strategy in VR. Zhao stressed that the summary strategy reduced learners' perceived success in interactive video which provided less-immersive learning experience [16]. In the experiment, the learners must write down the summarization of the VR lesson segment just viewed on a paper, which perhaps reduce the immersive learning experience of VR learning. Thus, it may the reason of the worse learning performance.

Concerning learning processes, although the VR+ST group and the $\mathrm{VR}+\mathrm{S}$ group induced more positive emotion, the $\mathrm{VR}+\mathrm{ST}$ group $(d=0.96)$ had a more impact on generative 
learning than the latter $(d=0.60)$. As mentioned above, the Self-Testing strategy provided the guiding function which reduced the difficulty perception of the VR lesson, then induced more positive emotions. We also found that the $\mathrm{VR}+\mathrm{ST}$ group gave more motivation, but the $\mathrm{VR}+\mathrm{S}$ group did not.

Emotion always a factor affecting memory. There was a broadening effect that students induced positive emotions during learning because available cognitive resources increased for getting information. In other words, the positive emotion not only takes good advantage of the cognition resources available but also facilitates the process of generative learning. Shortly, the superiority of the VR+ST group in positive emotion (as measured by emotion recognition technology) compared with the VR group translated into improvements in generative learning (selection-organization-integration) on immediate retention or delay-retention tests.

\section{B. Theoretical Contributions}

The present research contributes to the Self-Testing strategies used in VR chemical leaning on ICAML by using emotion recognition technology. As Rutherford stressed, "changes in emotional state influence higher cognition." [17]. By answering the questions of Self-Testing, learners induced positive emotion and motivation on VR learning that directly makes them pay attention to key elements of the VR lesson and make connections between the questions and chemical knowledge presented in VR, then results in a positive impact on memory performance.

However, the results also showed that the immediate memory weakened when learners used the generative summary strategy during a VR video. Social-Cognitive Theory stressed that perceived performance always influenced motivation, emotion, and task selection [18]. During the process of summarizing, learners who learn in less-immersive learning condition may be aware of the lack of immersion [19]. As a result, the generative summary strategy effect in VR is that lower perceived immersion students get influence mood, motivation, and attention to important information, finally, result in the poor performance of immediate retention test.

Unfortunately, we did not have measures to assess the immersion that learner perceived. Shortly, lack of the perceived immersion using the summary strategy in VR learning may impact mood and motivation, furthermore the generative learning process, which is a limitation that could be explored in future study.

However, learners used Self-Testing strategy in VR lesson, which asked them to write down the answer to the questions after the VR lesson segment, it also provided less-immersive learning condition, we did not find that learners immediate memory weakened but become stronger. Does the superiority of positive emotion induced by Self-Testing strategy make up for the disadvantages of less-immersive condition? It also a direction that could be study in the future.

\section{Practical Contributions}

This study points to the potential of the Self-Testing generative strategy that initiating deep cognitive processing (i.e., inducing positive affect and motivation to the learning material and making connections between emotion and cognition) during learning VR, which improve learners' immediate memory and long-term memory.

It should highlight that adding summarization into VR learning may diminish the memory, although the positive emotions arose. In short, if the aim is to memorize chemical, we suggest that the teacher gives priority to the Self-Testing strategy when students learn.

\section{LIMITATIONS AND FUTURE DiRECTIONS}

Based on the experiment, we believe that adding the Self-Testing into chemical VR lesson promise positive emotions and stronger memory, but Summarization may have the opposite effect. The current study provides evidence concerning the cognitive processes of generative strategy during learning, which generally are on ICAML.

However, understanding the connection between positive affect and immersion perceived in Summary strategy and Self-Testing strategy remains a central challenge. As the universal media to teach, the video also should be compared to VR video with a generative strategy on memory.

\section{CONFLICT OF INTEREST}

The authors declare no conflict of interest.

\section{AUTHOR CONTRIBUTIONS}

Wenya Yang conducted the research, analyzed the data; Wenya Yang and Xue Wang wrote the paper; all authors had approved the final version.

\section{REFERENCES}

[1] C. Y. Shih, "A virtual walk through London: culture learning through a cultural immersion experience," Computer Assisted Language Learning, vol. 28, no. 5, pp. 407-428, 2015.

[2] Y. Zhou, S. Ji, T. Xu, and Z. Wang, "Promoting knowledge construction: A model for using virtual reality interaction to enhance learning," Procedia Computer Science, vol. 130, pp. 239-246, 2018.

[3] G. Makransky and G. B. Petersen, "Investigating the process of learning with desktop virtual reality: A structural equation modeling approach," Computers \& Education, vol.134, pp. 15-30, 2019.

[4] Y. Cheng and S. H. Wang, "Applying a 3D virtual learning environment to facilitate student's application ability - The case of marketing," Computers in Human Behavior, vol. 27, no. 1, pp. 576-584, 2011.

[5] J. Parong and R. E. Mayer, "Learning science in immersive virtual reality," Journal of Educational Psychology, 2018.

[6] J. Leder, T. Horlitz, P. Puschmann et al., "Comparing immersive virtual reality and powerpoint as methods for delivering safety training: Impacts on risk perception, learning, and decision making," Safety Science, vol. 111, pp. 271-286, 2019.

[7] G. Makransky, S. Borre-Gude and R. E. Mayer, "Motivational and cognitive benefits of training in immersive virtual reality based on multiple assessments," Journal of Computer Assisted Learning, vol. 35, no. 6, pp. 691-707, 2019.

[8] O. A. Meyer, M. K. Omdahl, and G. Makransky, "Investigating the effect of pre-training when learning through immersive virtual reality and video: A media and methods experiment," Computers \& Education, vol. 140, 2019.

[9] L. Fiorella and R. E. Mayer, "Eight ways to promote generative learning," Educational Psychology Review, vol. 28, no. 4, pp. 717-741, 2016.

[10] J. Zhao, L. Lin, J. Sun, and Y. Liao, "Using the summarizing strategy to engage learners: Empirical evidence in an immersive virtual reality environment," The Asia-Pacific Education Researcher, no. 1, 2020. 
[11] A. A. Darabi, D. W. Nelson, and S. Palanki, "Acquisition of troubleshooting skills in a computer simulation: Worked example vs. conventional problem solving instructional strategies," Computers in Human Behavior, vol. 23, no. 4, pp. 1809-1891, 2007.

[12] J. L. Plass and U. Kaplan, "Emotional design in digital media for learning - ScienceDirect," Emotions, Technology, Design, and Learning, pp. 131-161, 2016.

[13] D. Watson, L. A. Clark, and A. Tellegen, "Development and validation of brief measures of positive and negative affect: the PANAS scales," Journal of Personality and Social Psychology, vol. 54, no. 6, pp. 1063-1988, 1988.

[14] J. D. Laird et al., "Remembering what you feel: The effects of emotion on memory," Journal of Personality \& Social Psychology, vol. 42, no. 4, pp. 646-657, 1982.

[15] C. M. Chen and H. P. Wang, "Using emotion recognition technology to assess the effects of different multimedia materials on learning emotion and performance," Library \& Information Science Research, vol. 33, no. 3, pp. 244-255, 2011.

[16] J. Zhao, L. Lin, J. Sun, and Y. Liao, "Using the Summarizing Strategy to Engage Learners: Empirical Evidence in an Immersive Virtual Reality Environment," The Asia-Pacific Education Researcher, no. 1, 2020.

[17] H. Rutherford and A. K. Lindell, "Thriving and surviving: Approach and avoidance motivation and lateralization," Emotion Review, vol. 3, no. 3, pp. 333-343, 2011.

[18] D. A. Cook and A. R. Artino, "Motivation to learn: An overview of contemporary theories," Medical Education, vol. 50, no. 10, pp. 997-1014, 2016.
[19] J. Zhao, L. Lin, J. Sun, and Y. Liao, "Using the Summarizing Strategy to engage learners: Empirical Evidence in an Immersive Virtual Reality Environment," The Asia-Pacific Education Researcher, no. 1, 2020.

Copyright $(92021$ by the authors. This is an open access article distributed under the Creative Commons Attribution License which permits unrestricted use, distribution, and reproduction in any medium, provided the original work is properly cited (CC BY 4.0).

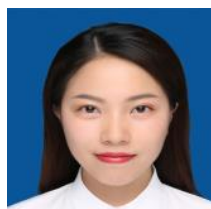

Yang Wenya is a postgraduate student in Tianjin Normal University. Her research looks into multimedia learning and digital media education.

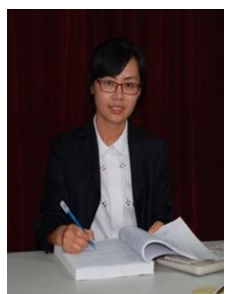

Wang Xue is an associate professor and master's supervisor of Educational Technology at Tianjin Normal University. She is a member of the Association for Educational Communications and Technology (AECT) and a partner of the Texas Center for Education Technology (TCET). Her research looks into Linguistics for Multimedia Design (LMD), Multimedia Learning and digital education resource optimization design and application. 